\title{
The Effect of Web-Based Aerobic Exercise on Working Memory in Older Women: the Importance of Being Active in the Covid- 19 Pandemic Period
}

\section{Maryam Noruztabar ${ }^{1}$, Mohammad Jalilvand ${ }^{*}$}

${ }^{1}$ Department of Physical Education and Sports Science, Kermanshah Branch, Islamic Azad University, Kermanshah, Iran

${ }^{2}$ Department of Physical Education and Sport Sciences, Hamedan Branch, Islamic Azad University, Hamedan, Iran

\section{ABSTRACT}

Introduction: The prevalence of the Covid-19 virus has affected the participation of the elderly in physical activity because restrictions have forced them to stay at home. This study aimed was to investigate the effect of eight weeks web-based aerobic exercise on working memory in older women. Materials and Methods: This study was quasi-experimental study. The statistical population in this study was all elderly women in Kermanshah, Iran. Thirty inactive elderly women who participated in the research voluntarily were randomly divided into experimental and control groups. Before the start of the training protocol Participants' working memory were measured using the N-Back test. The experimental group performed their exercises, which included web-based aerobic exercises for eight weeks and three sessions per week. To test the research hypotheses analysis of covariance was used in SPSS 23 software. Results: The results showed that web-based aerobic exercise had a significant effect on working memory of Kermanshah elderly women. Conclusion: Using web-based aerobic exercises can improve working memory in older women. Therefore, due to the limitations of movement caused by the Covid-19 pandemic and importance of the elderly being active for maintain cognitive health, it is recommended that the elderly use the web-based aerobic exercise.

Keywords:
1. Memory
2. Women
3. COVID-19

*Corresponding Author: Mohammad Jalilvand

Email:jalilvandmohammad@iauksh.ac.ir 


\section{تاثير تمرينات ايروبيك مبتنى بر وب بر حافظة كارى زنان سالمند: اهميت فعال بودن در دوره همهَيرى كوويد-19}

مريم نوروز تبار'، محمد جليلوند؟ّ

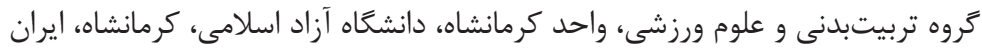

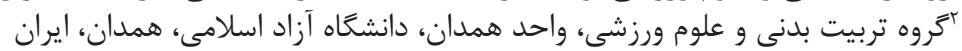

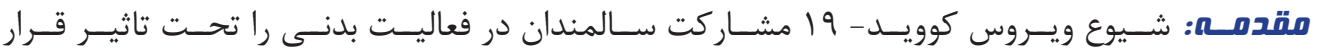

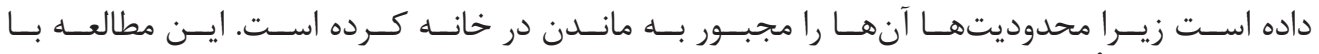

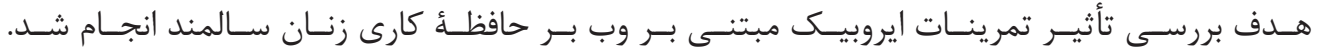

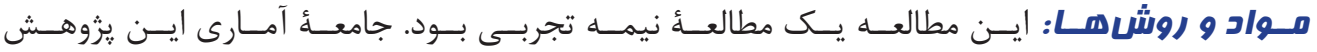

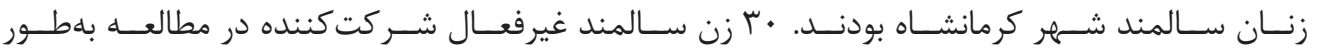

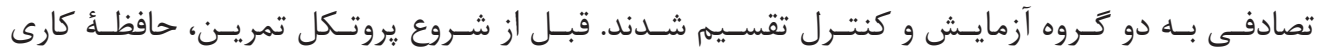

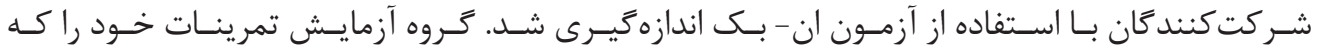

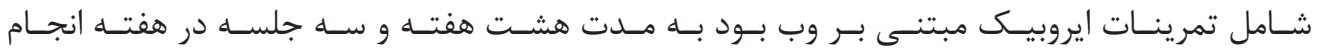

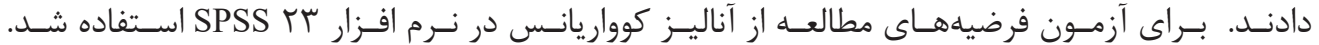

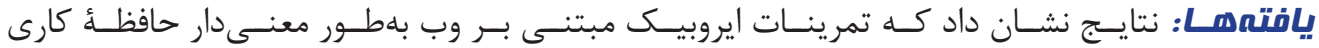

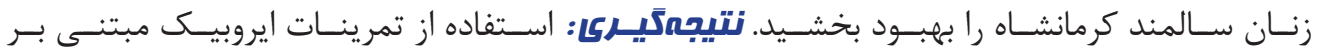

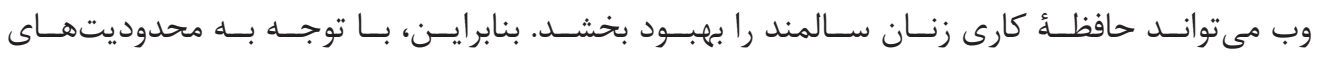

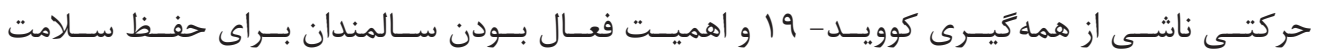

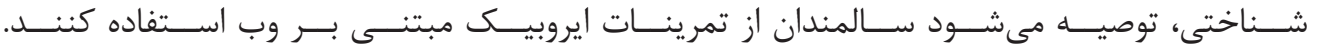

: 1- حافظه

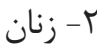

19-

*ويسنده مسئول: محمد جليلوند

يست الكترونيك: jalilvandmohammad@iauksh.ac.ir 


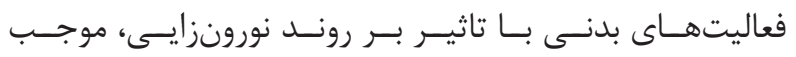

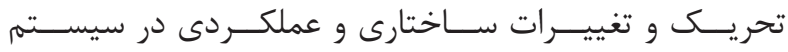

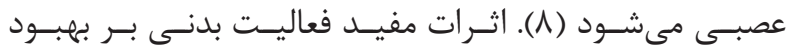

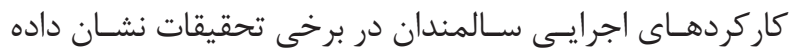

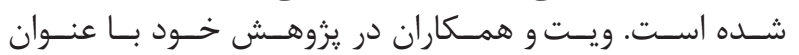

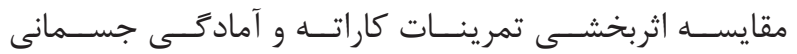

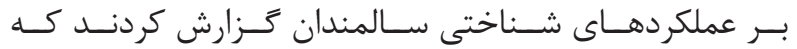

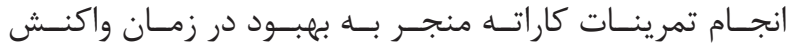

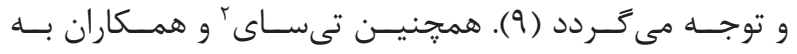

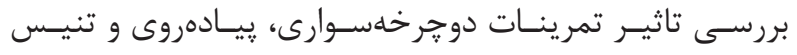

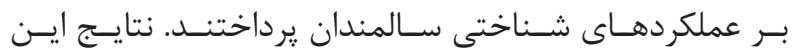

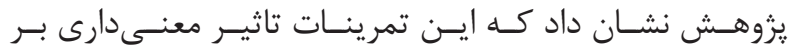

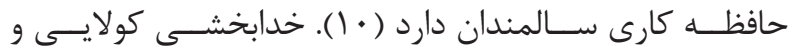

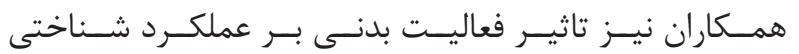

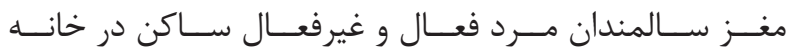

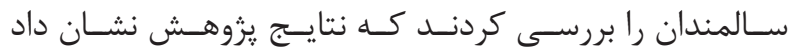

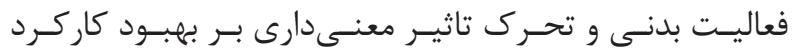

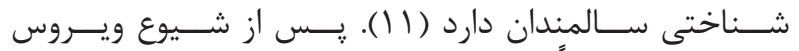

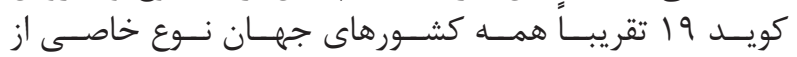

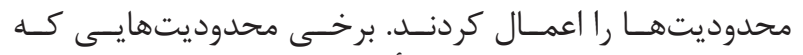

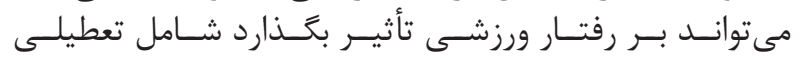

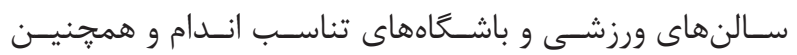

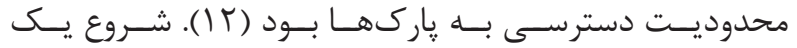

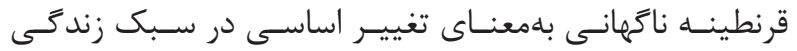

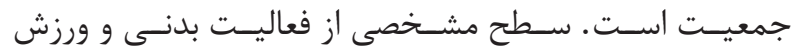

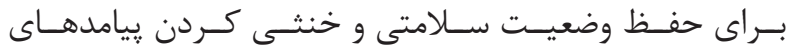

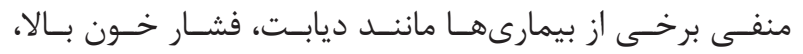

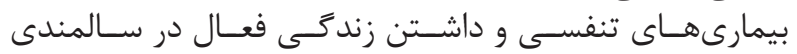

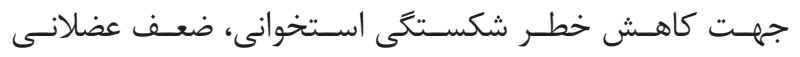

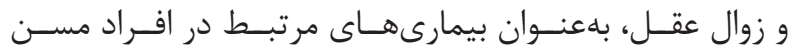

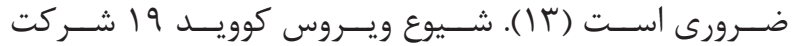

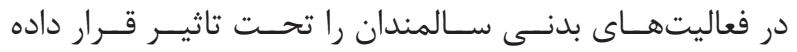

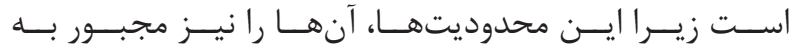

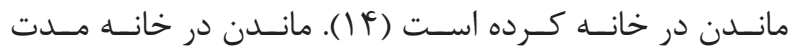

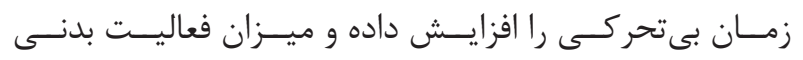

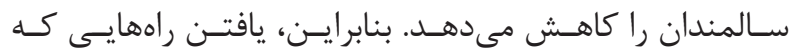

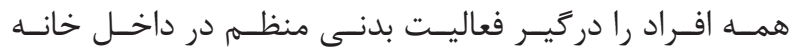

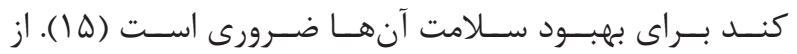

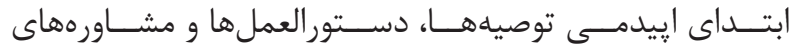

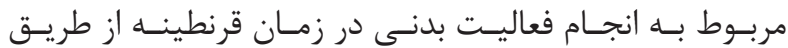

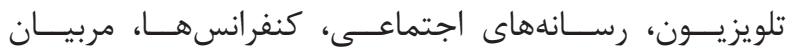

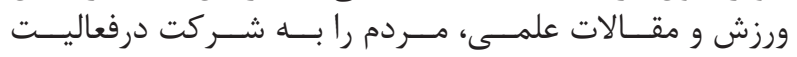

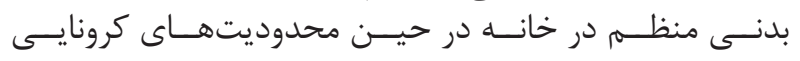

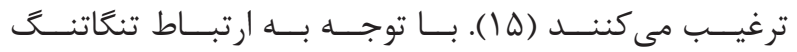

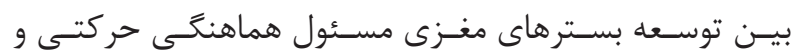

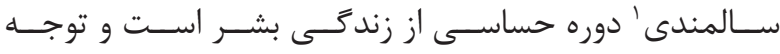

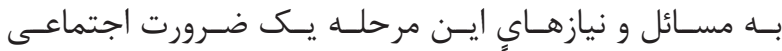

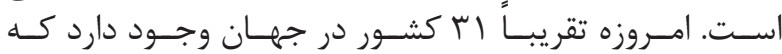

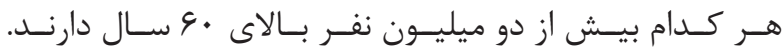

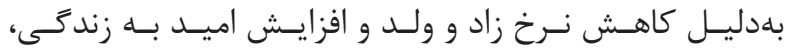

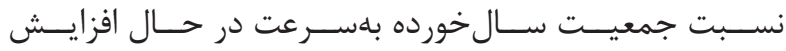

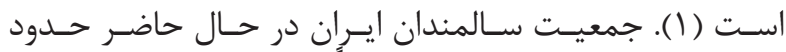

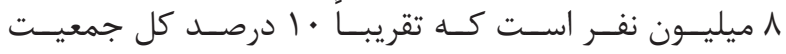

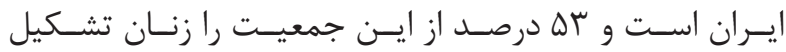

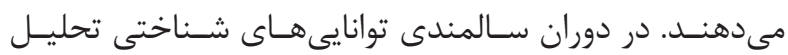

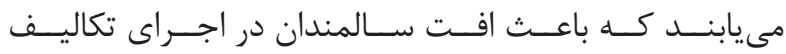

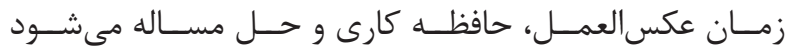

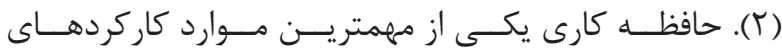

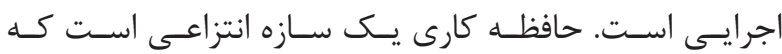

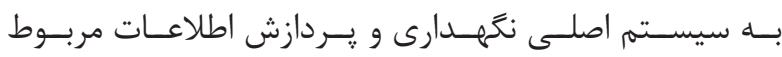

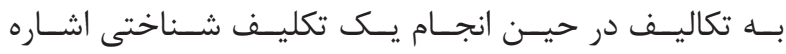

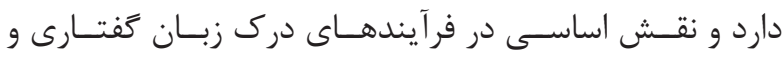

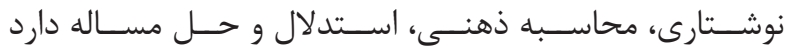

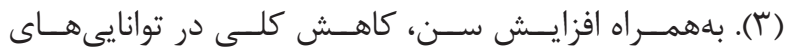

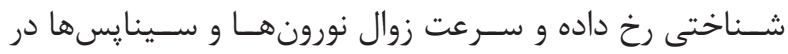

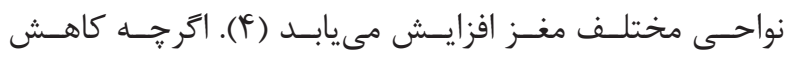

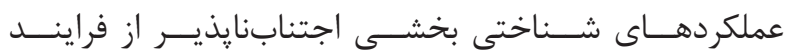

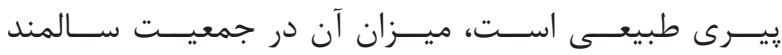

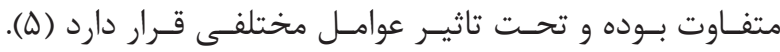

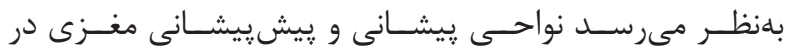

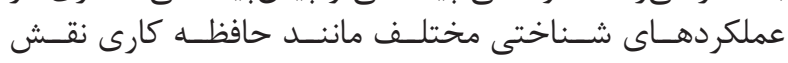

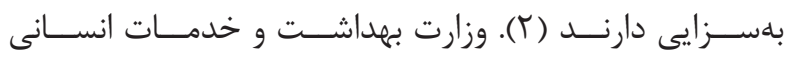

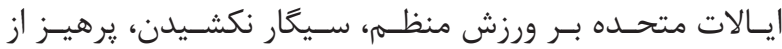

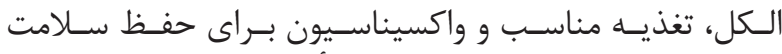

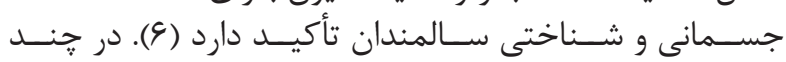

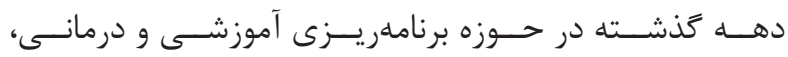

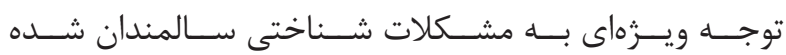

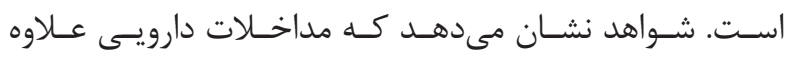

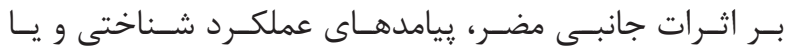

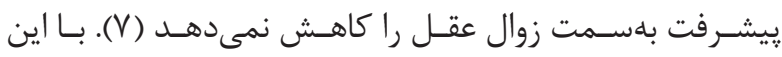

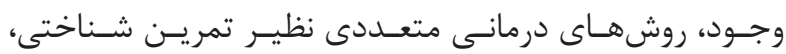

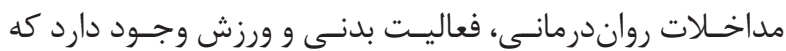

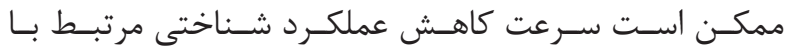

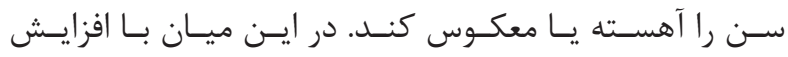

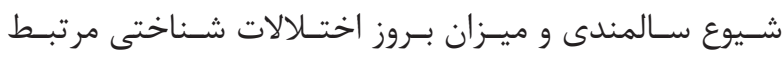

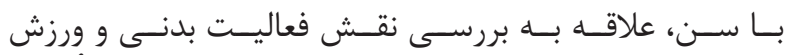

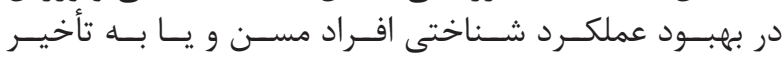

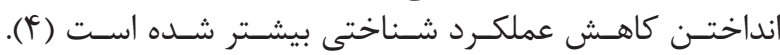




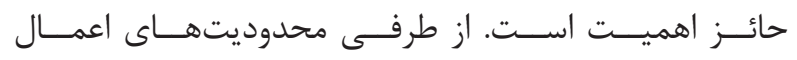

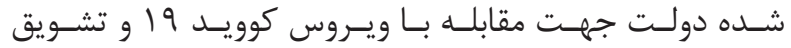

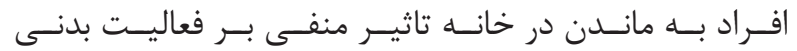

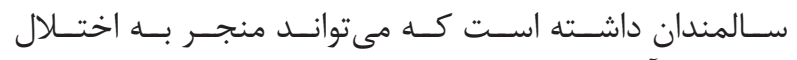

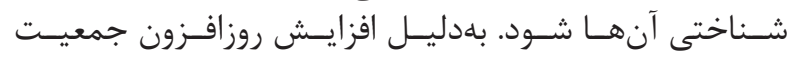

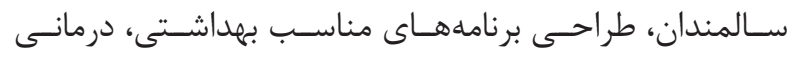

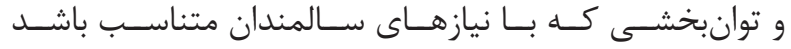

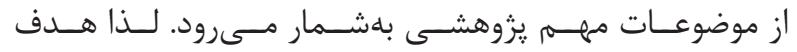

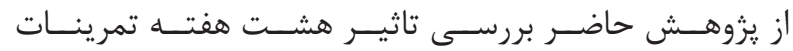

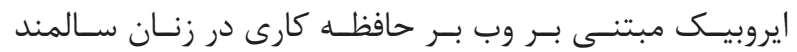

بـود.

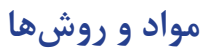

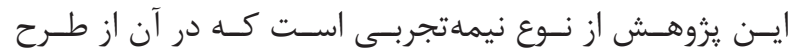

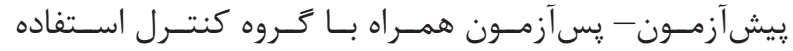

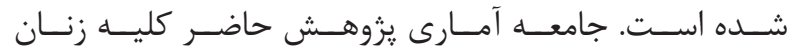

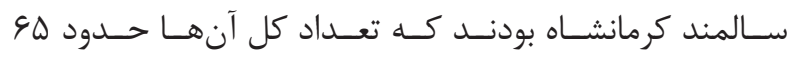

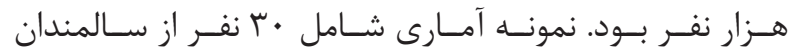

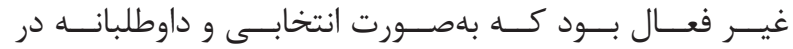

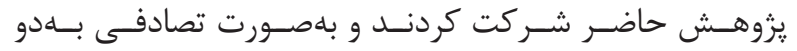

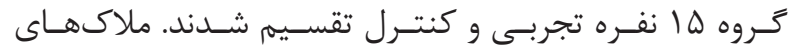

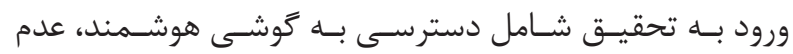

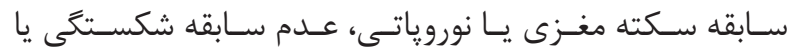

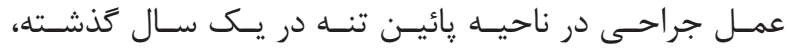

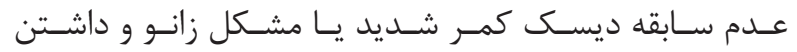

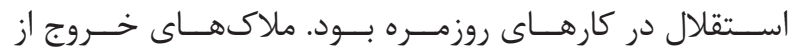

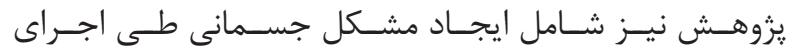

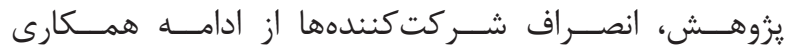

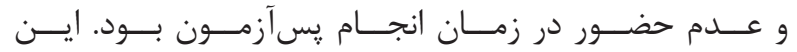

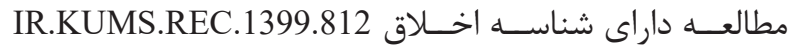

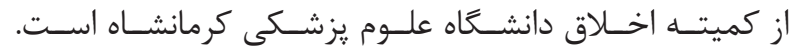

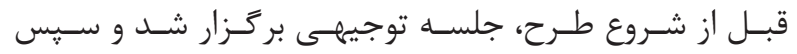

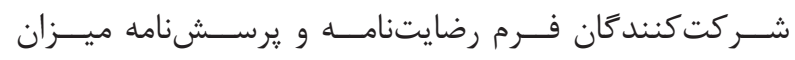

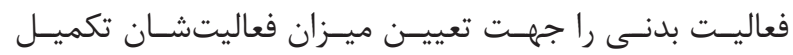

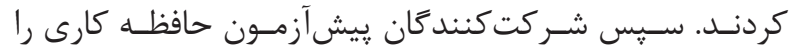

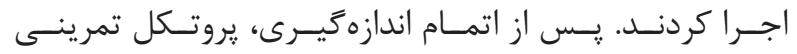

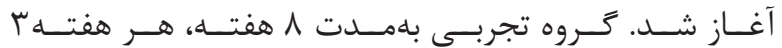

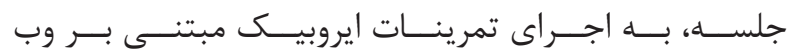

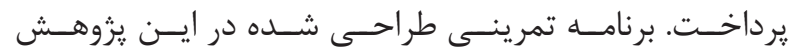

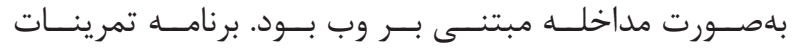

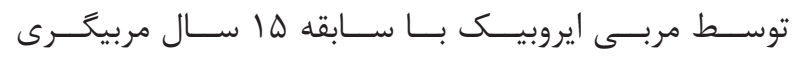

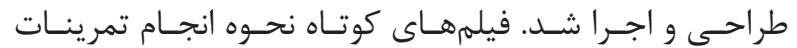

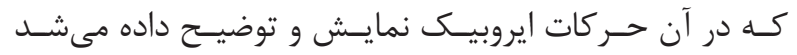

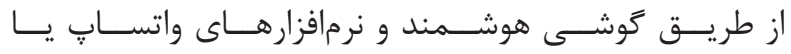

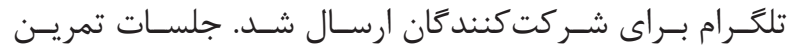

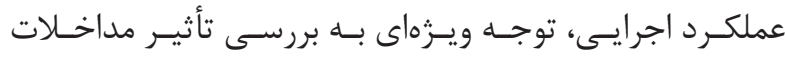

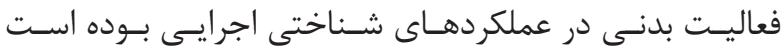

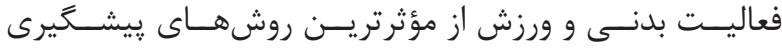

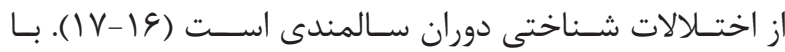

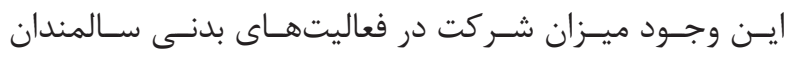

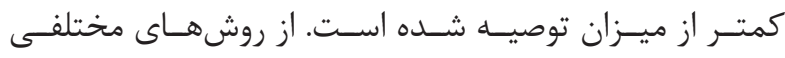

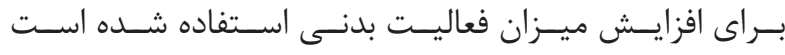

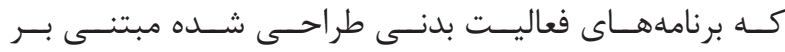

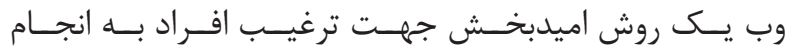

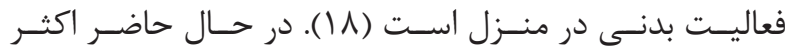

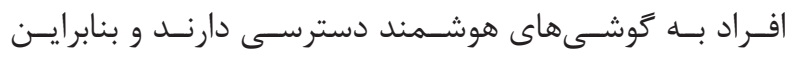

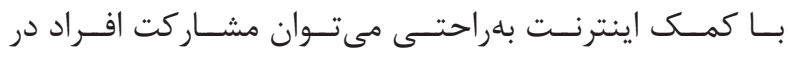

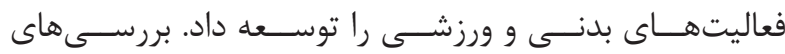

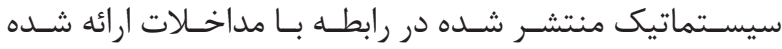

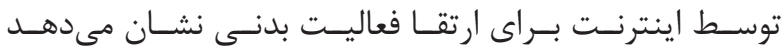

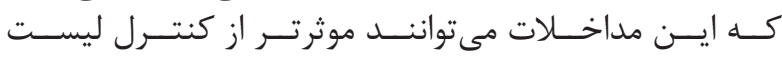

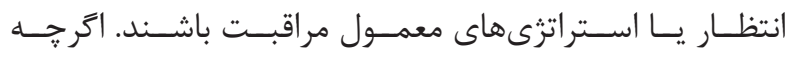

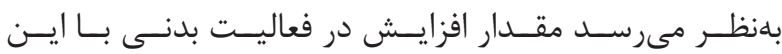

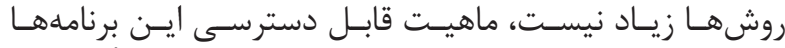

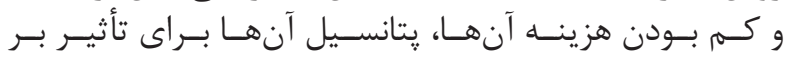

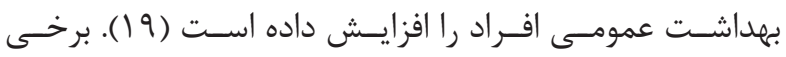

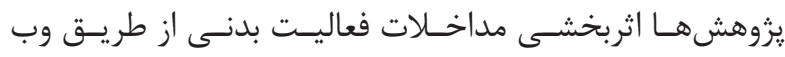

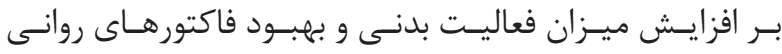

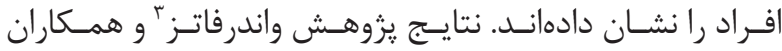

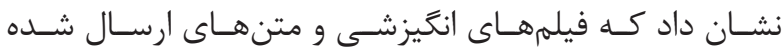

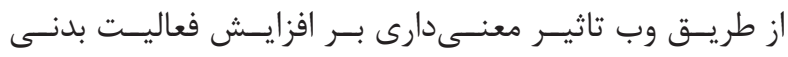

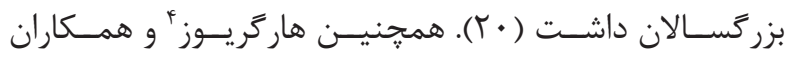

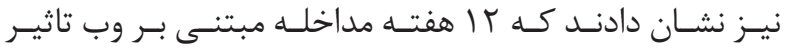

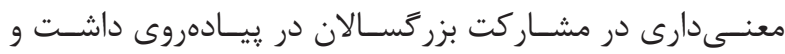

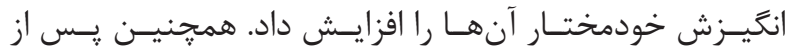

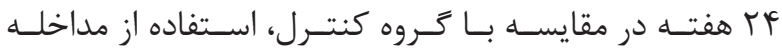

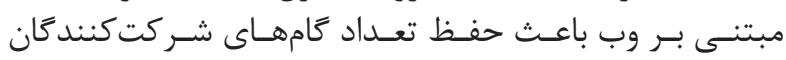

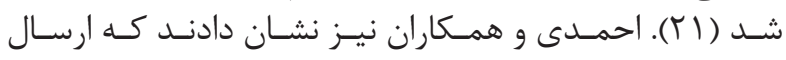

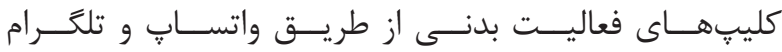

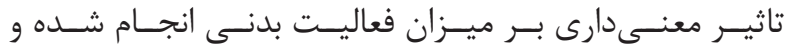

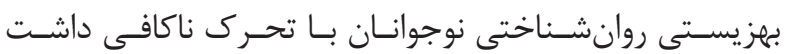

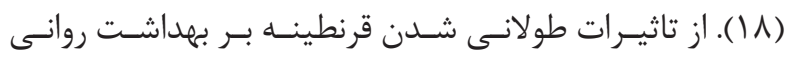

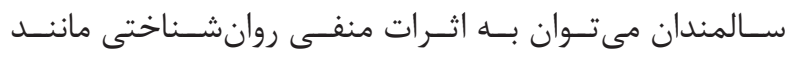

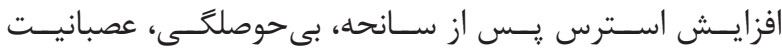

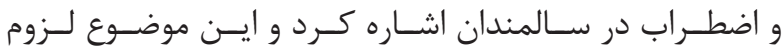

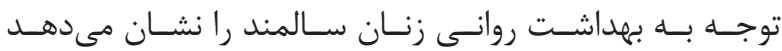

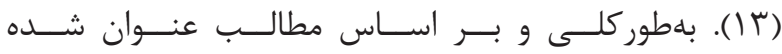

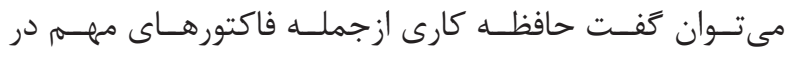

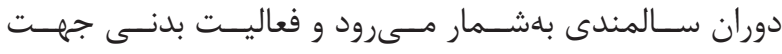

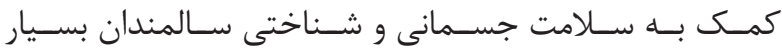


تحقيـق، دنبالـهاى از محركىهــاى ديـدارى بـود كـهـ بهصـورت

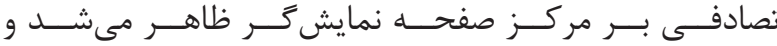

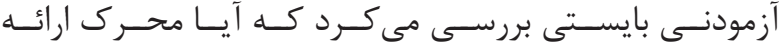

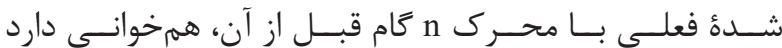

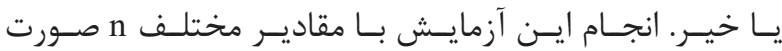

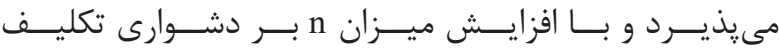

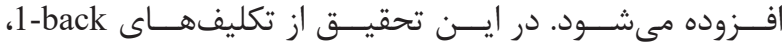

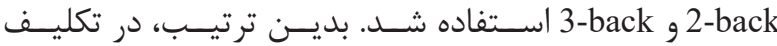

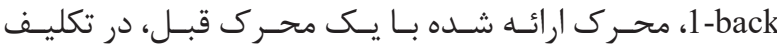
2-back 3-back

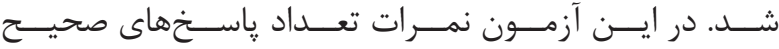

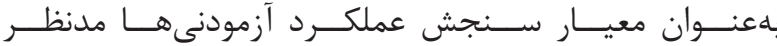

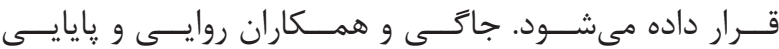

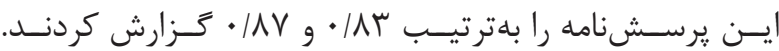

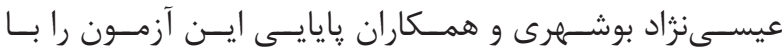

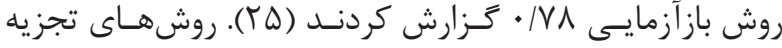

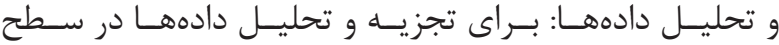

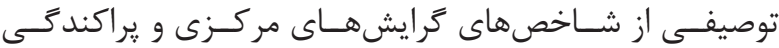

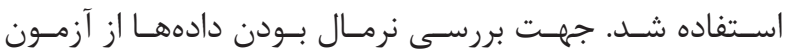

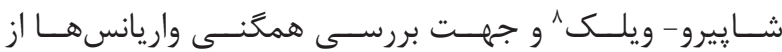

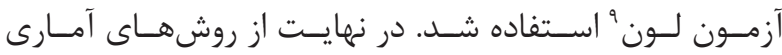

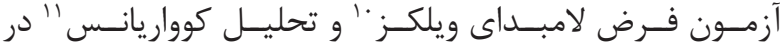

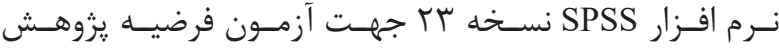

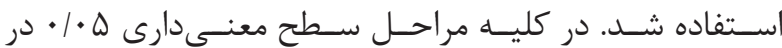

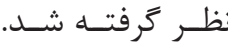

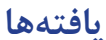

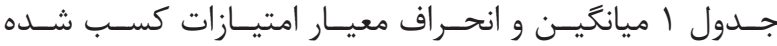

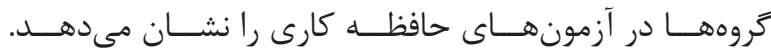

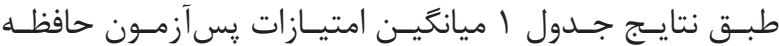

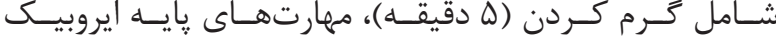

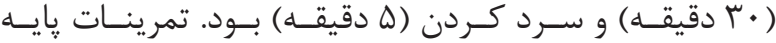

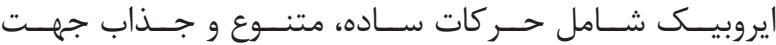

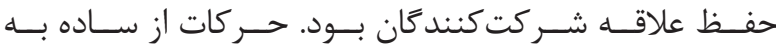

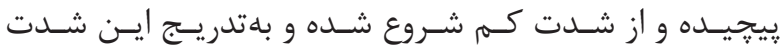

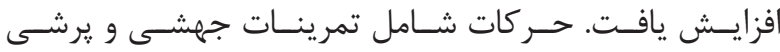

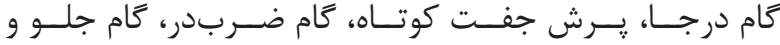

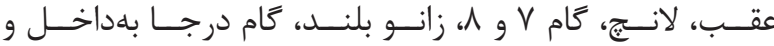

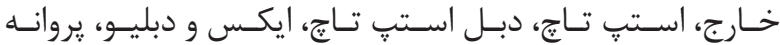

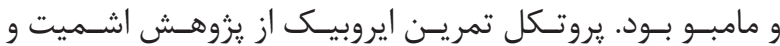

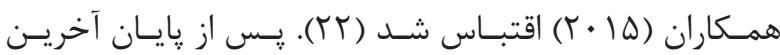

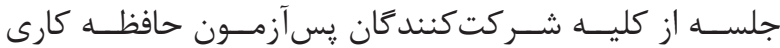

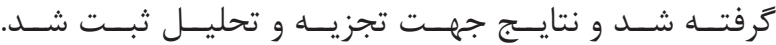

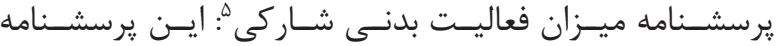

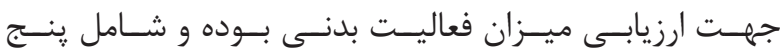

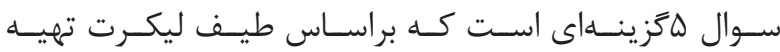

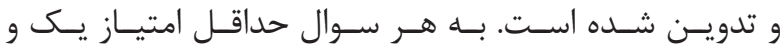

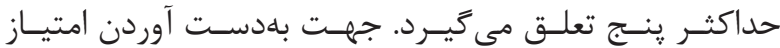

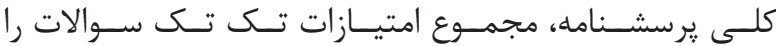

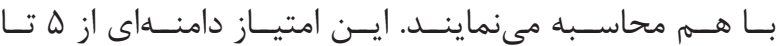

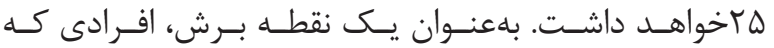

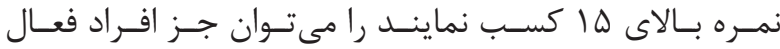

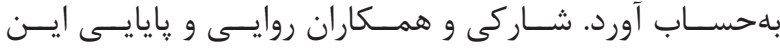

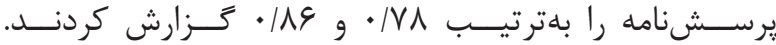

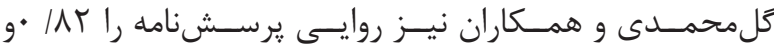

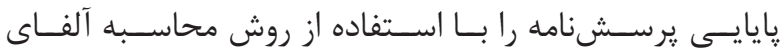

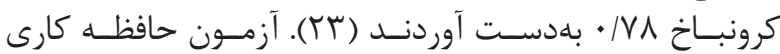

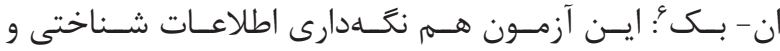

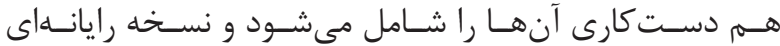

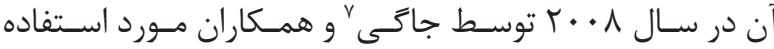

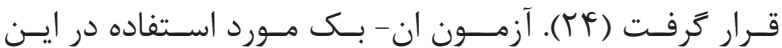

جدول 1- ميانگَين و انحراف معيار امتيازات حافظهٔ كارى در در دو گروه در بِيشآزمون و پِّآزمون

\begin{tabular}{|c|c|c|c|c|c|}
\hline \multicolumn{2}{|c|}{ پِ آزمون } & \multicolumn{2}{|c|}{ يِش آزمون } & \multirow{2}{*}{ تروه } & \multirow{2}{*}{ متغير } \\
\hline انحراف معيار & مياثگين & أحراف معيار & مياتخين & & \\
\hline r/QT & $G F / M$ & $F / \Delta V$ & $\Delta V / G \bar{G}$ & آزمايش & \multirow{2}{*}{ ان بك | } \\
\hline F/Tr & $\Delta \varphi / 1 Y$ & F/DT & $\Delta F / T r$ & كنترل & \\
\hline $1 / 90$ & FQ/KT & $r / \cdot G$ & rN/Dr & آزمايش & \multirow{2}{*}{ ان بك r } \\
\hline$r / \cdot r$ & rq/4q & $r / T)$ & rN/TG & كنترل & \\
\hline T/K & $r \cdot 19$. & $1 / 9 \wedge$ & $1 \omega / 9$. & آزمايش & \multirow{2}{*}{ ان بك r } \\
\hline $1 / \wedge \Delta$ & $19 / 1$. & $1 / 94$ & $1 Q / V \cdot$ & كنترل & \\
\hline
\end{tabular}

${ }^{5}$ Sharki Physical Activity Questionnaire

${ }^{6}$ Working Memory N-Back

${ }^{7}$ Jaeggi

${ }^{8}$ Shapiro-Wilk
${ }^{9}$ Leven test

${ }^{10}$ Wilkes's lambda hypothesis test

${ }^{11}$ Analysis of covariance 


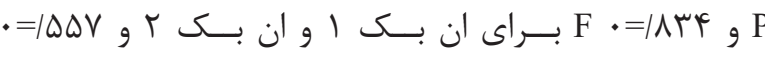

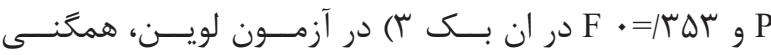

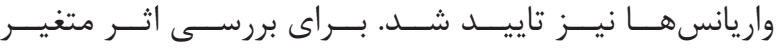

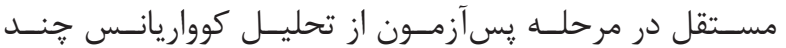

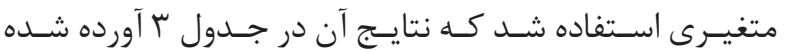

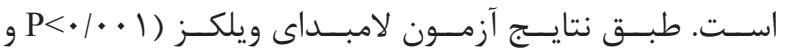

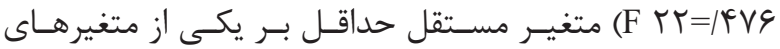

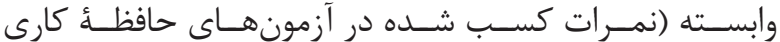

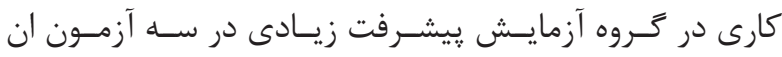

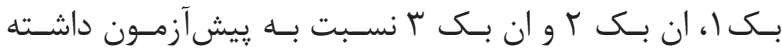

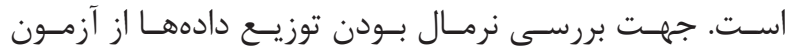

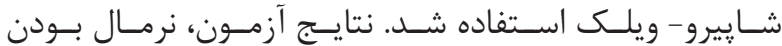

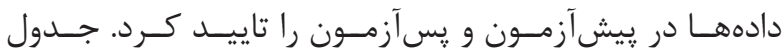

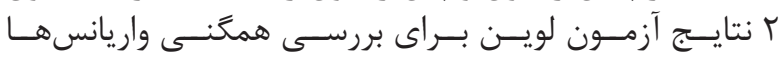

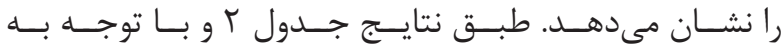

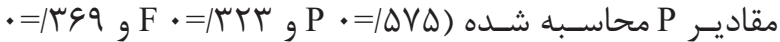

جدول r- نتايج آزمون لوين در مورد بيش فرض تساوى واريانسها

\begin{tabular}{|c|c|c|c|c|}
\hline \multicolumn{4}{|c|}{ لوين } & \multirow{2}{*}{ متغير } \\
\hline $\mathbf{P}$ & F & درجه آزادى r & درجهُ آزادى 1 & \\
\hline$\cdot \mid \Delta \vee \Delta$ & אזT/. & $r \Lambda$ & 1 & ان بك ا \\
\hline . T\&9 & - /AFY & TA & 1 & ان بك ץ \\
\hline$\cdot \mid \Delta \Delta V$ & $\cdot \mid r \Delta r$ & ru & 1 & ان بك ץ \\
\hline
\end{tabular}

جدول بـ- آزمون فرض لامبداى ويلكز براى تأييد فرض جند متغيره

\begin{tabular}{|c|c|c|c|c|c|c|}
\hline مجذور اتا & سطح معنى دارى & درجه آزادى & F & تخمين & آزمون & متغير \\
\hline$\cdot|V G|$ & $\cdot 1 \cdot \cdot 1$ & r & TY/FVG & $\cdot|V \varphi|$ & رديابى گيلايى & \multirow{3}{*}{ كروه } \\
\hline$\cdot|V \varphi|$ & $\cdot 1 \cdot \cdot 1$ & r & YY/FVG & . rrqq & ويلكز لامبدا & \\
\hline$\cdot|V \&|$ & $\cdot 1 \cdot \cdot 1$ & r & TY/FVG & $r / 19 \mu$ & اثر هاتلينگ & \\
\hline - $|V G|$ & $\cdot 1 \cdot \cdot 1$ & r & TY/FVG & $r / 19 \mu$ & \multicolumn{2}{|c|}{ بزرگترين ريشه روى } \\
\hline
\end{tabular}

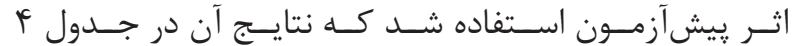

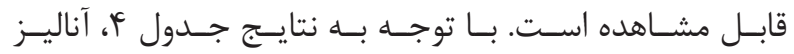

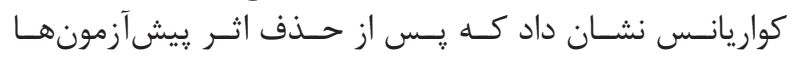

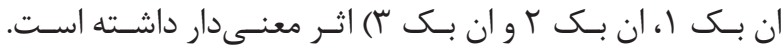

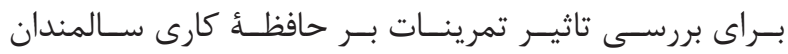

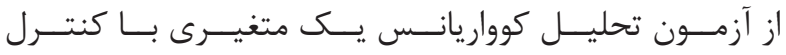

جدول F- نتايج حاصل از تحليل كوواريانس جهت مقايسٔ نمرات يس آزمون در متغير حافظة كارى

\begin{tabular}{|c|c|c|c|c|c|c|c|}
\hline مجذور اتا & معنى دارى & $\mathbf{F}$ & مياتين مجنورات & درجه آزادى & مجموع مجذورات & اثر & متغير \\
\hline .1994 & $.1 . .1$ & $\Delta H / A G$ & rАQ/\% & 1 & rیQ/r & يِيشآزمون & \multirow{3}{*}{ ان بك ا } \\
\hline \multirow[t]{2}{*}{$\cdot 109$} & $\cdot 1 . .1$ & rN/AD & $r|\cdot| r \mid$ & 1 & $r|\cdot| r \mid$ & گروه & \\
\hline & & & $D / f \mid$ & TV & $\mid f 9 / 1 \pi$ & خطا & \\
\hline.$/ 1 \mathrm{~V}$ & $.1 \cdot r \varepsilon$ & $D / D F$ & $r \cdot 1 \Lambda 9$ & 1 & $r \cdot 1 \Lambda 9$ & بيش آزمون & \multirow{3}{*}{ ان بك r } \\
\hline \multirow[t]{2}{*}{$\cdot|G Y|$} & $.1 . .1$ & FE/TY & TFE/. F & 1 & $r|q| \cdot F$ & تروه & \\
\hline & & & $\Delta / \Delta G$ & TV & $10 \cdot / r$. & خطا & \\
\hline$\cdot / \pi 9 \Lambda$ & $.1 . .1$ & $|Q / V|$ & $|f| / r$. & 1 & $|f| / T$. & بيش آزمون & \multirow{3}{*}{ ان بك r } \\
\hline$\cdot 19 \cdot 0$ & $\cdot 1 . .1$ & $f \mid / r$. & $1 \cdot N / \mu$. & 1 & $1 \cdot N / \mu$. & تروه & \\
\hline & & & $r / 9 Y$ & TV & $V \cdot / V q$ & خطا & \\
\hline
\end{tabular}




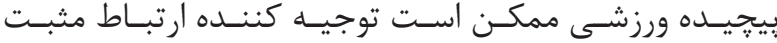

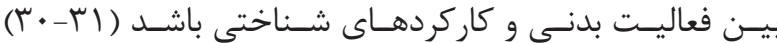

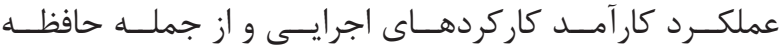

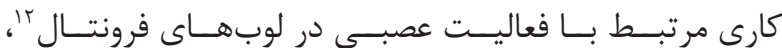

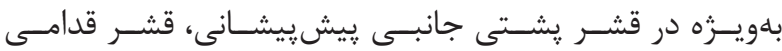

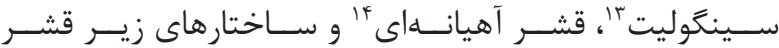

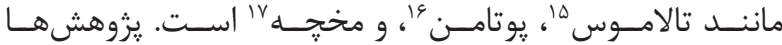

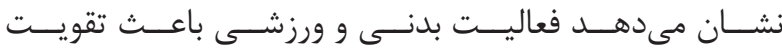

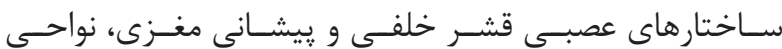

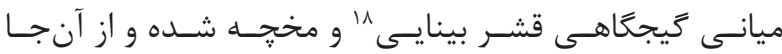

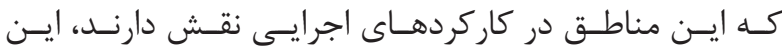

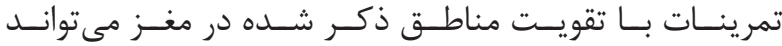

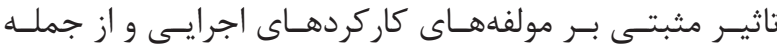

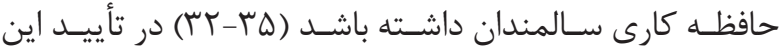

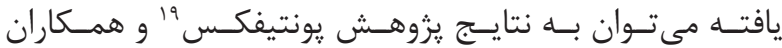

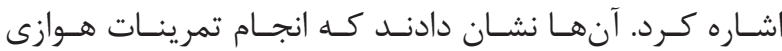

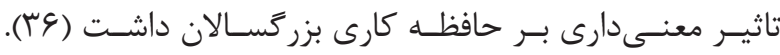

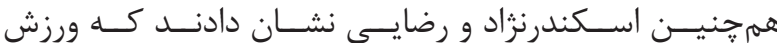

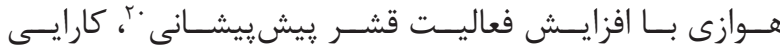

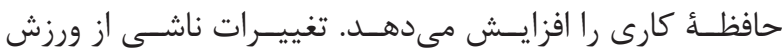

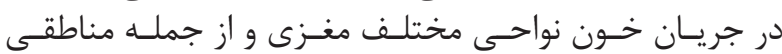

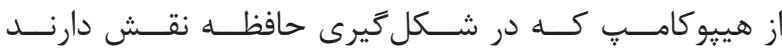

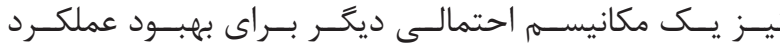

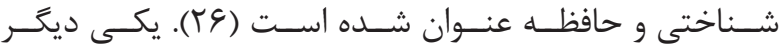

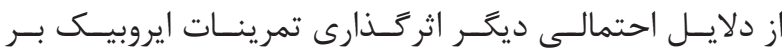

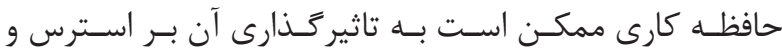

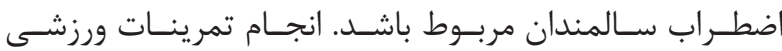

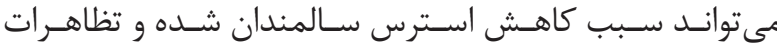

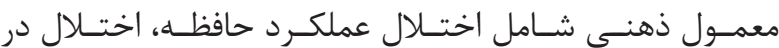

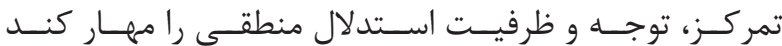

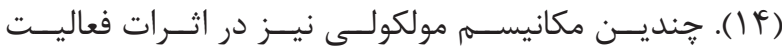

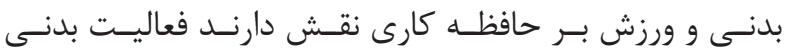

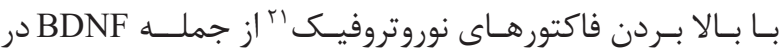

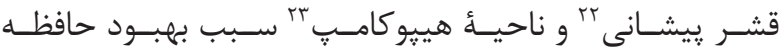

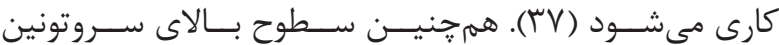

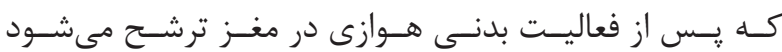

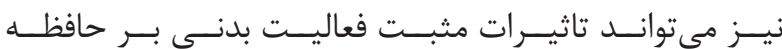

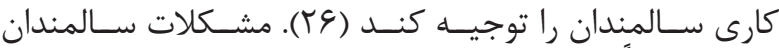

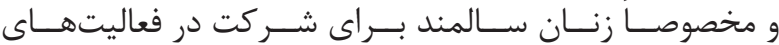

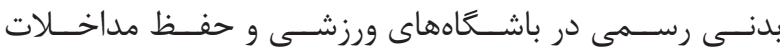

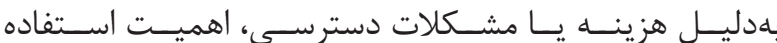

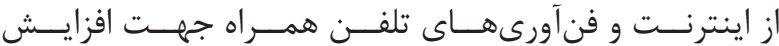

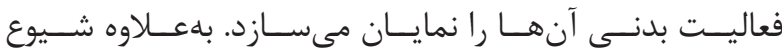

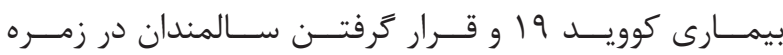

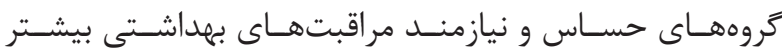

\footnotetext{
${ }^{12}$ Frontal lobes

${ }^{13}$ Cingulate

${ }^{14}$ Parietal cortex

${ }^{15}$ Thalamus

${ }^{16}$ Putamen

${ }^{17}$ Cerebellum
}

تفــاوت معنـى دارى در يـس آزمــون نمــرات حافظــــ كارى در

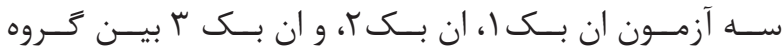

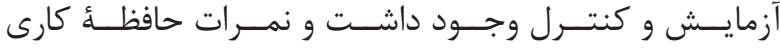

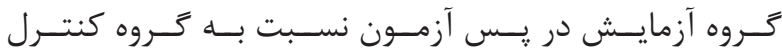

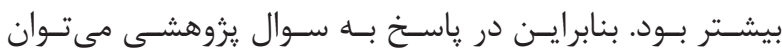

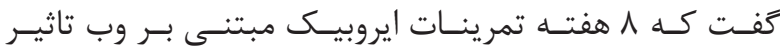

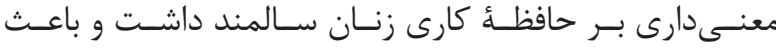

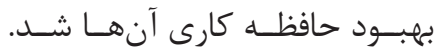

بحث و نتيجه كَيرى

هــدف يروهــش حاضــر بررســى تاثيــر \ هفتــهـ تمرينــات

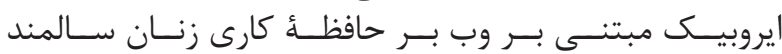

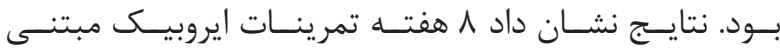

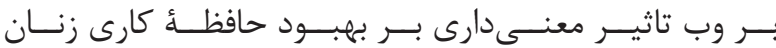

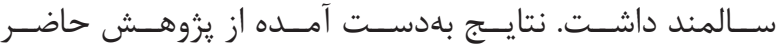

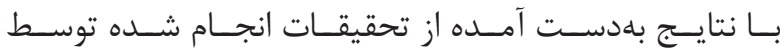

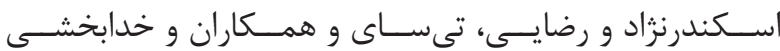

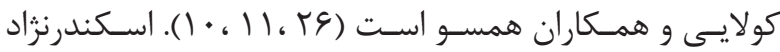

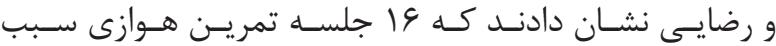

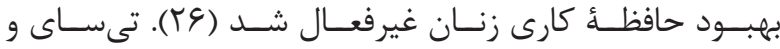

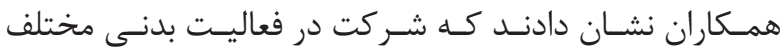

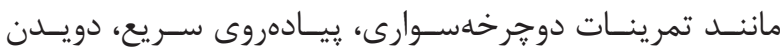

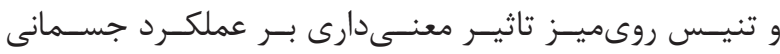

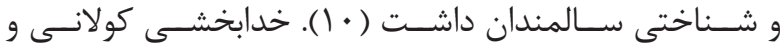

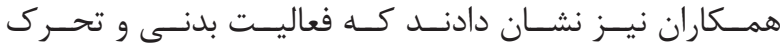

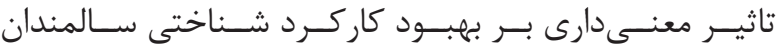

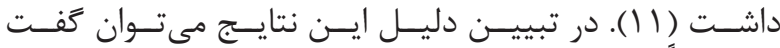

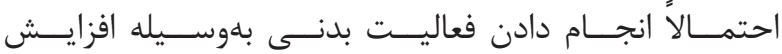

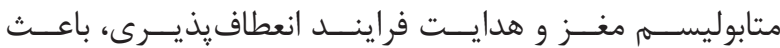

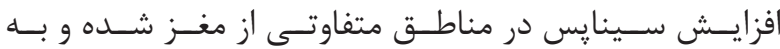

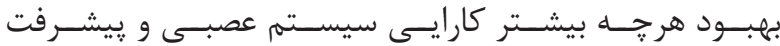

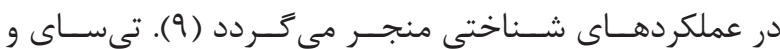

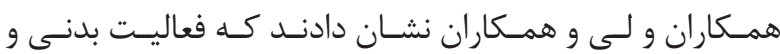

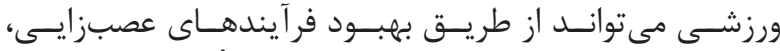

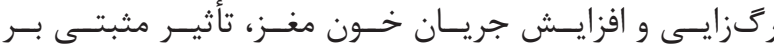

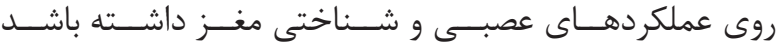

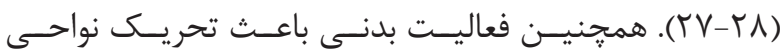

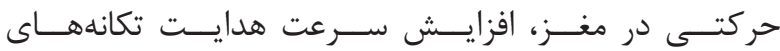

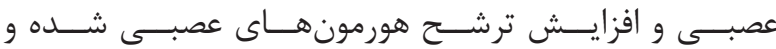

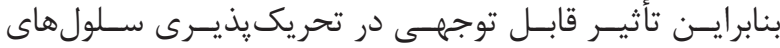

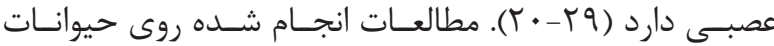

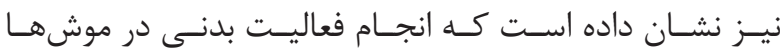

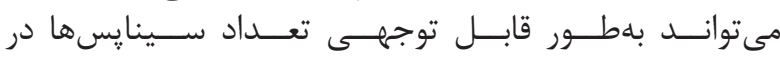

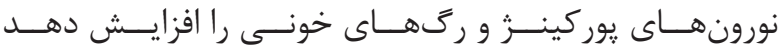

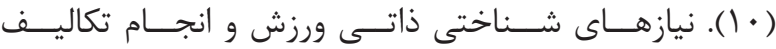

${ }^{18}$ Temporal areas of the visual cortex

${ }^{19}$ Pontifex

${ }^{20}$ Prefrontal

${ }^{21}$ Neurotrophic factors

${ }^{22}$ Forehead cortex hippocampus

${ }^{23}$ Hippocampus 
از محدوديتهــاى يزوهــش حاضــر مــى تـوان بـــه محسـدود

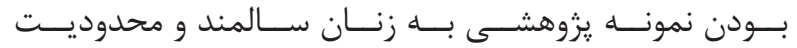

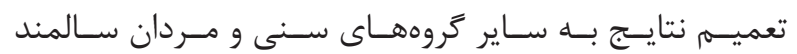

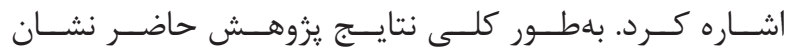

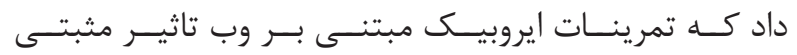

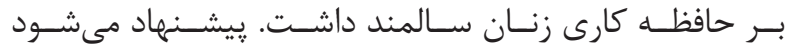

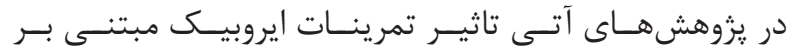

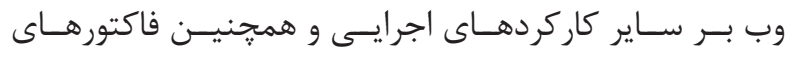

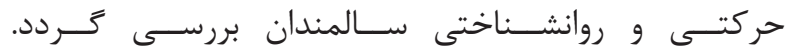

1. Zaninotto P, Batty GD, Stenholm S, Kawachi I, Hyde M, Goldberg M, et al. Socioeconomic inequalities in disability-free life expectancy in older people from England and the United States: A cross-national population-based study. The Journals of Gerontology: Series A. 2020;5)75 ):.906-13

2. Peymannia B, Bitarafan L, Hosseini A. Evaluation and comparison of executive functions and false memory in the elderly. Shenakht. 2019; 6(5): 108-17.

3. Baddeley A. Working memory: looking back and looking forward. Nature Reviews Neuroscience. $\quad 2003 ; \quad 4(10)$ 8 $\quad$ 829-39.

4. Shabani F, Esmaeili A, Salman Z. Effectiveness of Different Intensities of Acute Resistance Exercise on Working Memory of the Elderly. Aging Psychology. 2017; 3(1): 55-67.

5. Anderson LA, McConnell SR. Cognitive health: an emerging public health issue. Alzheimer's \& dementia: the journal of the Alzheimer's Association. 2007; 3(2): S70-S3.

6. Gwinnutt JM, Verstappen SMM, Humphreys JH. The impact of lifestyle behaviours, physical activity and smoking on morbidity and mortality in patients with rheumatoid arthritis. Best Practice \& Research Clinical Rheumatology. 2020; 3(1): 101562.

7. Farina N, Llewellyn $\mathrm{D}$, Isaac $\mathrm{MG}$, Tabet $\mathrm{N}$. Vitamin E for Alzheimer's dementia and mild cognitive impairment. The Cochrane database of systematic reviews. 2017; 1(1): 2854.

8. Najian A, Nejati V. Effectiveness of motor based cognitive rehabilitation on improvement of sustained attention and cognitive flexibility of children with ADHD. The Scientific Journal of Rehabilitation Medicine. 2018; 6(4): 1-2.

9. Witte K, Kropf S, Darius S, Emmermacher P,
و از سـويى محدوديتهــاى اعمــال شـده توســـ دولتهــا در

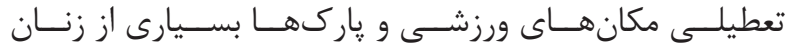

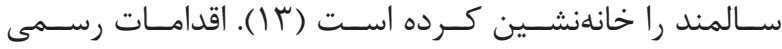

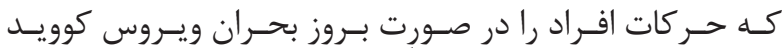

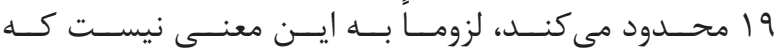

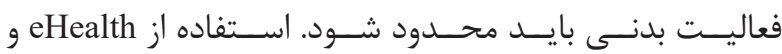

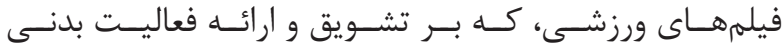

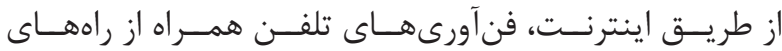

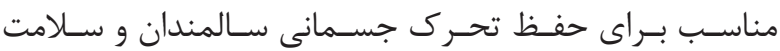

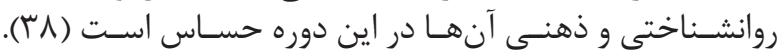

منابع

Böckelmann I. Comparing the effectiveness of karate and fitness training on cognitive functioning in older adults - a randomized controlled trial. Journal of Sport and Health Science. 2016; 5(4): 484-90.

10. Tsai C-L, Pan C-Y, Chen F-C, Tseng Y-T. Openand closed-skill exercise interventions produce different neurocognitive effects on executive functions in the elderly: a 6-month randomized, controlled trial. Frontiers in Aging Neuroscience. 2017; 9: 294.

11. Khodabakhshi-koolaee A, Sabzi S, Shahdadi H, Mohamadi F. The Comparison of Brain Cognition Function between Active and Inactive Elderlies Male in Nursing Home (A Case-control Study in Tehran). Health. 2017; 4(4): 302-9.

12. Brand R, Timme S, Nosrat S. When pandemic hits: Exercise frequency and subjective well-being during COVID-19 pandemic. Frontiers in psychology. 2020; 11: 2391.

13. Jiménez-Pavón D, Carbonell-Baeza A, Lavie CJ. Physical exercise as therapy to fight against the mental and physical consequences of COVID-19 quarantine: Special focus in older people. Progress in cardiovascular diseases. $2020 ; 63(3)$ : 386.

14. Smirmaul BPC, Chamon RF, de Moraes FM, Rozin G, Moreira ASB, de Almeida R, et al. Lifestyle Medicine During (and After) the COVID-19 Pandemic .American Journal of Lifestyle Medicine. 2021; 15(1): 60-7.

15. Barwais FA. Physical Activity at Home During the COVID-19 Pandemic in the Two Most-affected Cities in Saudi Arabia. The Open Public Health Journal. 2020; 13(1): 470-6.

16. Leonard HC. The impact of poor motor skills on perceptual, social and cognitive development: the case of developmental coordination disorder. Frontiers in psychology. 2016; 7: 311.

17. Jalilvand M. The effectiveness of physical 


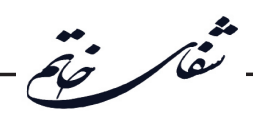

activity with motor-cognitive approach on executive function in children with Attention Deficit/Hyperactivity Disorder 2020.; 8(2): 17-26.

18. Ahmadi A, Aghdasi M, Ahmadi M. Effects of Web-Based Physical Activity Interventions on Physical Activity and Well-being in Adolescents with Insufficient Physical Activity. Quarterly Journal of Health Psychology. 2018; 6(24): 53-68.

19. Tate DF, Lyons EJ, Valle CG. High-tech tools for exercise motivation: use and role of technologies such as the internet, mobile applications, social media, and video games. Diabetes Spectrum. 2015; 28(1): 45-54.

20. Van der Fels IM, te Wierike SC, Hartman E, ElferinkGemserMT,SmithJ, VisscherC. Therelationshipbetween motor skills and cognitive skills in 4-16 year old typically developing children: A systematic review. Journal of science and medicine in sport. 2015; 18(6): 697-03.

21. Hargreaves EA, Mutrie N, Fleming JD. A web-based intervention to encourage walking (StepWise): pilot randomized controlled trial. JMIR research protocols. 2016; 5(1): e14.

22. Schmidt M, Jäger K, Egger F, Roebers CM, Conzelmann A. Cognitively engaging chronic physical activity, but not aerobic exercise, affects executive functions in primary school children: a group-randomized controlled trial. Journal of Sport and Exercise Psychology. 2015; 37(6): 575-91.

23. Golmohammadi B, Kashani V, Mokaberian M. Persian Psychometric properties of homework Self-Efficacy Scale for Everyday Elderly Activities. J Clin Psychol. 2015; 7(2): 78-89.

24. Jaeggi SM, Buschkuehl M, Jonides J, Perrig WJ. Improving fluid intelligence with training on working memory. Proceedings of the National Academy of Sciences. 2008; 105(19): 6829-33.

25. Isanejad bushehri s, dadashpur ahangar m, salmabadi $\mathrm{h}$, ashoori j, dashtbozorgi $\mathrm{z}$. The effect of computer games on sustain attention and working memory in elementary boy students with attention deficit / hyperactivity disorders. medical journal of mashhad university of medical sciences. 2016; 59(5): 311-21.

26. Eskandarnejad M, Rezaei F. The Effect of Aerobic Exercise on Neural Networks of Attention and Working Memory . The Neuroscience of Shefaye Khatam. 2018; 6(2): 31-40.

27. Tsai C-L, Chen F-C, Pan C-Y, Wang C-H,
Huang T-H, Chen T-C. Impact of acute aerobic exercise and cardiorespiratory fitness on visuospatial attention performance and serum BDNF levels. Psychoneuroendocrinology. 2014; 41: 121-31.

28. Li D, Huang C-J, Liu S-C, Chang K-H, Hung T-M. Exercise type relates to inhibitory and error processing functions in older adults. Aging, Neuropsychology, and Cognition. 2019; 26(6): 865-81.

29. Hollmann W, Strüder H. Brain, psyche and physical activity. Der Orthopade. 2000; 29(11): 948-56.

30. Ballester R, Huertas F, Molina E, Sanabria D. Sport participation and vigilance in children: Influence of different sport expertise. Journal of sport and health science. 2018; 7(4): 497-04.

31.JalilvandM, sourir. TheEffectivenessofMotorActivityBased Executive Function Training on Working Memory and Sustained Attention of Children with Attention Deficit/Hyperactivity Disorder. iricss. 2020;22(4): 87-98.

32. Von Hofsten C. An action perspective on motor development. Trends in cognitive sciences. 2004; 8(6): 266-72.

33. Diamond A. Close interrelation of motor development and cognitive development and of the cerebellum and prefrontal cortex. Child development. 2000; 71(1): 44-56.

34. Ramnani N. Frontal lobe and posterior parietal contributions to the cortico-cerebellar system. The Cerebellum. 2012; 11(2): 366-83.

35. Zwicker JG, Missiuna C, Boyd LAJJocn. Neural correlates of developmental coordination disorder: a review of hypotheses. Journal of child neurology. 2009; 24(10): 1273-81.

36. Pontifex MB, Hillman CH, Fernhall BO, Thompson KM, Valentini TA. The effect of acute aerobic and resistance exercise on working memory. Medicine \& Science in Sports \& Exercise. 2009; 41(4): 927-34.

37. Griffin ÉW, Mullally S, Foley C, Warmington SA, O’Mara SM, Kelly ÁM. Aerobic exercise improves hippocampal function and increases BDNF in the serum of young adult males. Physiology \& behavior. 2011; 104(5): 934-41.

38. Chen P, Mao L, Nassis GP, Harmer P, Ainsworth BE, Li F. Wuhan coronavirus (2019-nCoV): The need to maintain regularphysicalactivity whiletaking precautions. Journal of sport and health science. 2020; 9(2): 103. 